\title{
Development of a thin, internal superconducting polarisation magnet for the Polarised Target
}

\author{
Marcel Bornstein*, Hartmut Dutz, Stefan Goertz, Scott Reeve, Stefan Runkel \\ University Bonn, Germany \\ E-mail: bornsteinephysik.uni.bonn.de
}

In order to improve the figure of merit of double-polarisation experiments at CB-ELSA in Bonn, the Polarised Target is working on a new dilution refrigerator. For maximum polarisation of nucleons low temperatures and a high homogeneous magnetic field within the target area is needed. A thin, superconducting magnet is in development, which will create a continuous longitudinal magnetic field of $2.5 \mathrm{~T}$ and which will be used within the new refrigerator. The solenoidal geometry of this magnet uses two additional correction coils, placed at a well defined calculated position, for reaching the homogeneity criteria of $10^{-4}$ needed for the dynamic nuclear process. Practically, the superconducting wires as well as the correction coils have to be placed with maximum precision: Small fluctuations of the distance between the current loops can diminish the requested homogeneity. A second build prototype passes first tests and looks promising to fulfil the particular requirements.

XVIth International Workshop in Polarized Sources, Targets, and Polarimetry, PSTP2015,

14-18 September 2015,

Bochum, Germany

${ }^{*}$ Speaker. 


\section{Introduction}

For improving the figure of merit of double-polarisation experiments at CB-ELSA in Bonn the development of the Continuous Mode-Target has been introduced in 2001 [1]. One key element for this concept is a thin, high field polarisation magnet, which is placed within the refrigerating structure. With such a magnet the dynamic nucleon polarisation process (DNP) could be used during the measurement of the experiment. Hence the mean polarisation of the target could be close to maximum polarisation. This is much higher than the mean polarisation in the actual used Frozen-Spin-Mode [2]. In addition the complex handling of the huge detector components and external magnet is no longer required. There is no interruption of the experiment for re-polarising the target. The temperature of the target in the Continuous-Mode is about $300 \mathrm{mK}$ which is much higher than $30 \mathrm{mK}$ in the Frozen-Spin-Mode. More cooling power of the refrigerator is available at higher temperatures. Therefore a higher beam intensity could be accepted for the target.

\section{Boundary condition and design concept}

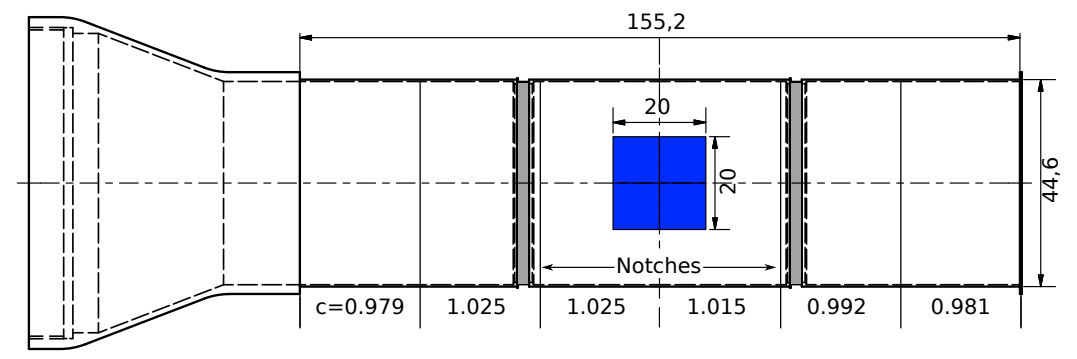

Figure 1: Technical drawing of an internal polarisation magnet. The target area is located in the magnetic center. Within this area the magnetic field must fulfil the homogeneity criteria. The grey filled areas indicate the notches. The six values on the bottom side are the factors $c$ used for the density calculation. Length-units are in $\mathrm{mm}$.

The geometric dimensions must be similar to the internal holding magnets[2], which are used in the Frozen spin mode. A technical drawing of the coil carrier can be seen in figure 1. The overall thickness of $2 \mathrm{~mm}$ should not be exceeded otherwise the detecting threshold will be raised by absorption of particles in the structure. For the DNP process a magnetic field of $2.5 \mathrm{~T}$ is needed. To fulfil this condition with respect to the thickness criteria a superconducting wire ${ }^{1}$ is used. The main field is created by a 6-layer solenoid with 586 windings per layer.

For polarising deuterons the inhomogeneity of the field within the target area must be smaller than $\Delta B=10^{-4}$. Because the field of a perfect solenoid is not sufficent $\left(\Delta B>10^{-3}\right)$, two correction coils are added. The homogeneity is very sensitive to any misalignment of these coils. In contrast to the Notched Solenoid [4] they are placed in the inner radius of the solenoid. Two notches in the carrier give maximum preciseness for positioning the coils (fig. 1). Hence this concept is called the Inverse Notched Solenoid. Each correction coil has 2.9 windings.

The whole magnet has an inductance of $160 \mathrm{mH}$ and a stored energy of $650 \mathrm{~J}$ at $2.5 \mathrm{~T}$. The wires are fixed to the carrier structure by a cryogenic epoxy glue in a wet wiring process.

\footnotetext{
${ }^{1}$ multifilament NbTi, Ø0.254 mm
} 


\section{Calculation}

\subsection{Biot-Savart-law}

For calculating the magnetic field of a solenoid and its correction coils the Biot-Savart-Law is used (eq. 3.1).

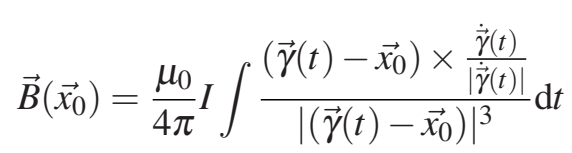

Figure 2 shows all necessary vectors for a certain path $\gamma(t)$. For this design the path is given by a loop parametrisation (eq. 3.2) with $r$ as the radius of each loop and $d$ as the effective diameter between two wires.

$$
\vec{\gamma}=\left(r \cos (t), r \sin (t), n \cdot d_{\text {eff }}\right)
$$

The product of the counting variable $n$ and the effective diameter $n \cdot d_{\text {eff }}$ gives the position of each loop. Due to the superposition principle the field of each loop may be added up to an overall field at a position $x_{0}$. To find the best position of the correction coils an algorithm loops over all possible positions and calculates the maximum field inhomogeneity (eq. 3.3) within the target area. The central field $B_{0}=B(\overrightarrow{0})$ is used here as the reference value.

$$
\Delta B\left(\overrightarrow{x_{0}}\right)=\frac{B\left(\vec{x}_{0}\right)-B_{0}}{B_{0}}
$$

The field inhomogeneity can be calculated to $\Delta B<0.4 \times 10^{-4}$ with the best position for the correction coils . Figure 3 shows the whole field map calculated for this case. The slight asymmetric structure comes from the fact that the magnetic centre does not coincides with the geometric centre. This is because of the orthocyclic winding scheme (see fig.4) in combination of a constant loop number for each layer.

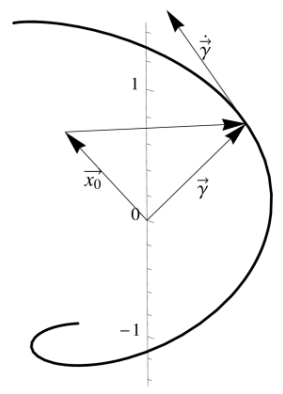

Figure 2: Electric current on a helictical path. The Biot-Savart-Law is used for calculating the magnetic flux density $B$ at the position $x_{0}$.

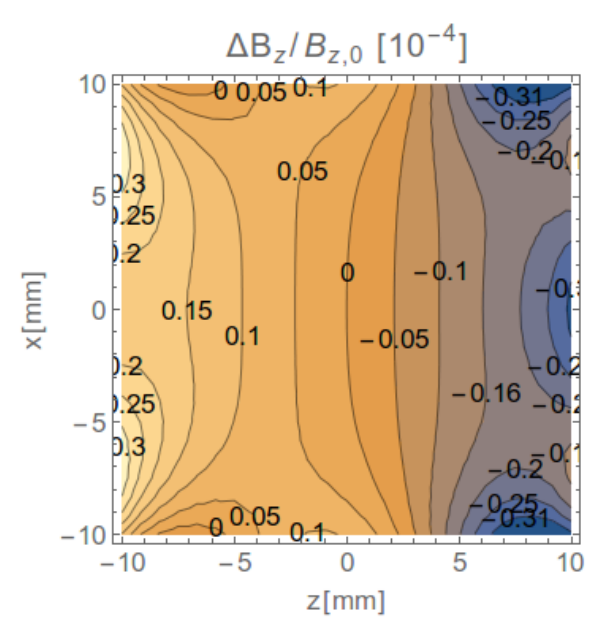

Figure 3: Field map of the target area. 


\subsection{Effective diameter}

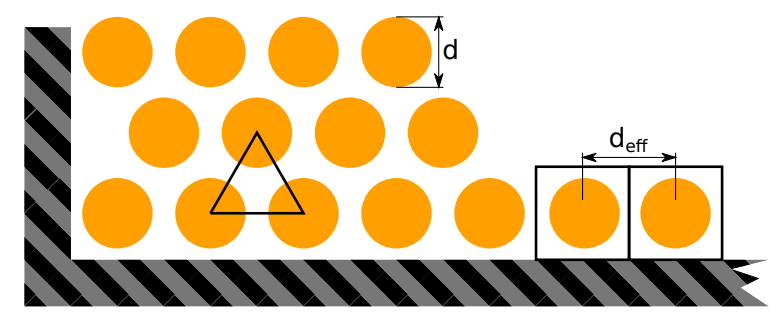

Figure 4: Schematical drawing of an orthocyclic wiring.

The effective diameter $d_{\text {eff }}$ is defined as the distance between one wire and its neighbour. Because there is some space filled up with glue it does not coincide with the true wire diameter $d$. As in figure 4 can be seen in orthocyclic wirings [5] the wire form a equilateral triangle with $d_{e f f}$ as edge length. The effective diameter is mainly depended by the glue's viscosity, the tensile stress and (lateral) pressing forces on the wire. The latter is caused by the hand tool which applies the glue onto the surface and the efficiency of the wire guiding mechanism.

\section{First prototype}

\subsection{Estimation of the field homogeneity}

A first prototype of an internal polarisation magnet was produced and tested in liquid helium at a temperature of $4.2 \mathrm{~K}$ and a current of $65 \mathrm{~A} \mathrm{[3].} \mathrm{A} \mathrm{hall} \mathrm{probe} \mathrm{could} \mathrm{be} \mathrm{moved} \mathrm{along} \mathrm{the} \mathrm{central}$ axis of the magnet for measuring the magnetic field $B$. The data are plotted in figure 5 with $\mathrm{B}$ against the position $z$ on the magnet's central axis. As it can be seen there is significant discrepancy between the measured and the calculated field. A asymmetric structure with two local maxima can be seen near the position of the correction coils. From this data it is clear, that the criteria of homogeneity has not been reached.

An explanation for this behaviour can be found by a precise analysis of the wire position on the solenoid.

\subsection{Density calculation}

When calculating the magnetic field a homogeneous current density is assumed over the whole solenoid by determining the effective diameter as constant. In practice the effective diameter can vary during the wiring process. This lead to a local variation of the wire density which is equivalent to a local variation of the current density.

A simple model considering this effect is introduced in figure 1. The solenoid is split up into 6 parts, where the effective distance is modulated by a factor $c$. A factor $c<1$ means the effective diameter is reduced, the current density in this region is raised. As it can be seen in figure 5 this simple model can reproduce the measured asymmetric structure by varying the effective diameter only by about $2 \%$. Evidence for this behaviour could be found on the magnets surface with a microscope.

In Conclusion the local variation of the wire has to be reduced otherwise the homogeneity will be diminished. 


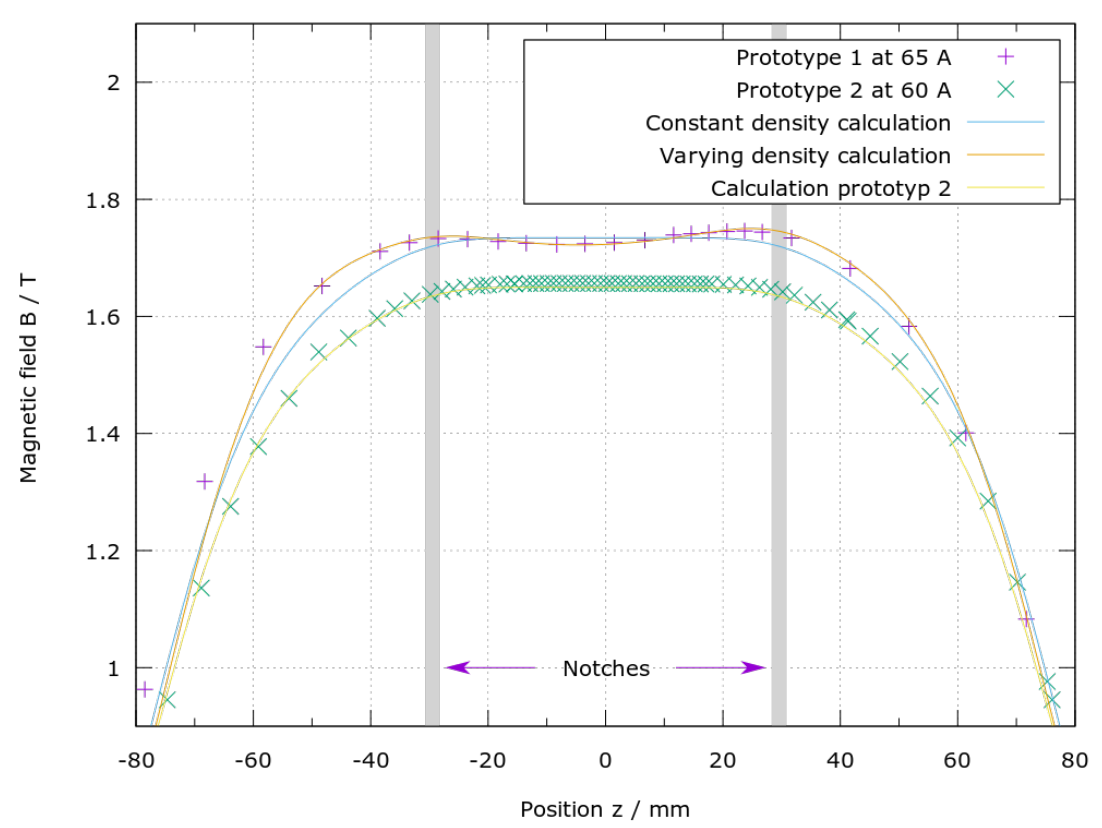

Figure 5: Comparision of the prototyp's field measurement and their calculations. The grey filled rectangles illustrate the position and size of the two correction coils.

\section{Second protoype}

The variation of the effective diameter could be reduced to a value better than $1 \%$ by improving the wiring mechanism. During the wiring process the actual wired loop has been guided with a constant pressing force toward its neighbouring loop. A second prototype could be build with this new wiring mechanism.

\subsection{Estimation of the field homogeneity}

\subsection{Field measurement}

The second prototype has been tested in liquid helium with a current of $60 \mathrm{~A}$. The data can be seen in figure 5. In contrast to the first prototype there is a flat plateau within the target area, which is as expected. The field inhomogeneity within the target area on the z-axis can be estimated only to $\Delta B \leq 10^{-3}$ with this setup caused by hall probe's uncertainties .

For a further test the NMR-signals of a polyethylen-sample in thermal equilibrium has been recorded. The cylindric sample with a diameter of $5 \mathrm{~mm}$ and a heigth of $22 \mathrm{~mm}$ could be moved on the z-axis. Figure 6 shows the normalised signals at different position. A frequency-shift of each peak was compensated by a adjusting the current/magnetic field. The field shift can be estimated by this adjustment with an uncertainty of $0.35 \mathrm{mT}$ around the centre field.

\section{Conclusion}

A thin, internal superconducting polarisation magnet for the Polarised Target is actually in development. A magnetic field of $2.5 \mathrm{~T}$ with a field inhomogeneity better than $10^{-4}$ within the 


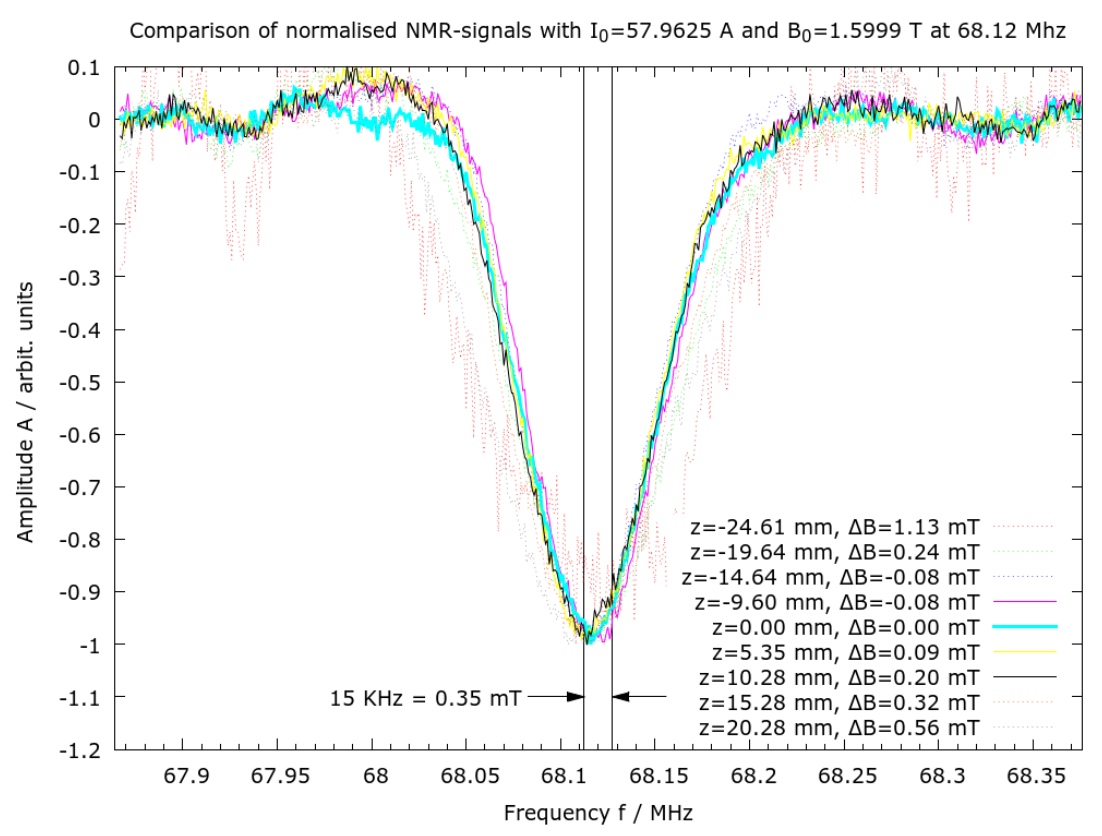

Figure 6: Normalised NMR-Signals of a polyethylen sample in thermal equilibrium in different positions within the magnet. All solid lines correspond to a position within the target area.

target area is necessary for the DNP process with Deuterons. The basic concept - the Inverse Notched Solenoid- works with a solenoid for the main field and two correction coils which are added in the inner radius. This gives an accurate positioning of these correction coils.

Tests with the first prototype showed that the field inhomogeneity can be influenced by the a local variation of the wire density in the solenoid. Therefore this variation has been reduced with an improved wiring meachnism. A second prototyp has been build and passed first tests: The field measurement on the z-axis showes the expected flat plateau within the target area. NMR-Signals of polyethylen in thermal equilibrium at $4.2 \mathrm{~K}$ could be seen with this magnet and indicates a sufficient homogeneity.

For the next step the magnet will be installed within a ${ }^{4} \mathrm{He}$-refrigerator. The maximum possible current will be determined at $1 \mathrm{~K}$. Addionally the magnet will be tested for dynamically polarizing a butanol-target.

\section{References}

[1] H. Dutz, , in proceedings of $16^{\text {th }}$ Int. Spin Phy. Symp., Trieste, Italy 2004, World Scientific (2005) 221

[2] C.Bratke, A New Frozen-Spin Target for the Measurement of Helicity Asymmetry of the Total Photoabsorption Cross-Section, PHD-Thesis, Bonn University, Bonn 2000

[3] M. Bornstein, Bau und Test eines kryostatinternen supraleitenden Polarisationsmagneten, Diploma-Thesis, Bonn University, Bonn 2013.

[4] Y. Iwasa, Case Studies in Superconducting Magnets, Second Edition, Springer US, New York 2009.

[5] E. Kallenbach, et al. Elektromagnete -Grundlagen, Berechnung, Entwurf, 4. Edition, Vieweg + Teubner Verlag, Wiesbaden, 2012. 